\title{
On the total curvature of a closed curve
}

\author{
By Shigeo SASAKI \\ (Received July 7, 1959)
}

\section{§ 1. Introduction.}

Let $x_{\alpha}(s)^{1)}$ be a parametric representation of a closed curve $C$ in Euclidean $n$-space $E_{n}$ in terms of its arc length $s$. If we denote the length of the curve by $L$, then $x_{\alpha}(s)$ 's may be regarded as periodic functions of period $L$ defined over $-\infty<s<+\infty$. When all functions $x_{\alpha}(s)$ are of class $C^{\prime \prime}$ we say that the curve is of class $C^{\prime \prime}$. Denoting the first curvature of $C$ by $k(s)(\geqq 0)$ we say that the integral

$$
K(C)=\int_{0}^{L} k(s) d s
$$

is the total curvature of the curve.

In 1929, W. Frenchel $[1,2]$ proved for $n=3$ the following theorem.

The total curvature of a closed curve of class $C^{\prime \prime}$ is greater than or equal to $2 \pi$. It is equal to $2 \pi$ when and only when the curve is a plane convex curve.

Different proofs were also given by H. Liebmann [3], B. Segre [4] and the theorem was generalized by K. Borsuk [5], H. Rutishauser and H. Samelson [6] to the $n$-dimensional case. M. I. Fary [7], J. W. Milnor [8] and R. H. Fox [9] studied the case where the closed curve is a knot.

The purpose of this paper is to sharpen Fenchel's theorem making use of $\mathrm{J}$. Douglas' theory on Plateau's problem [10] and of the Gauss-Bonnet's theorem.

The results of $\S 2,3$ and 4 seem to be known. However, their proofs, as far as I know, have never been published. So I write proofs completely. The main theorem in $\S 5$ seems to be new.

\section{. § 2. A canonical form of a minimal surface in a neihborhood of a singular point.}

First take the unit circle in a complex $w$-plane and denote the closed domain bounded by the unit circle by $W$. Let $F_{\alpha}(w), \alpha=1,2, \cdots, n$, be $n$ analytic functions defined on $W$ which are related by

$$
\sum_{\alpha=1}^{n} F_{\alpha}^{\prime 2}(w)=0,
$$

then the surface $S$ in $E_{n}$ defined by

$$
x_{\alpha}=\Re F_{\alpha}(w)
$$

is a minimal surface in the sense of Weierstrass, where $x_{\alpha}$ 's are rectangular cartesian coordinates in $E_{n}$. The minimal surface $S$ may have self-intersection, but we do not

1) In this paper we suppose that indices take the following ranges of values $\alpha=1,2, \cdots, n ; \quad a, b, c, d, e=1,2 ; \quad \rho, \sigma=1,2, \cdots, n-2$. 
regard self-intersection as a singularity. However, the point $\mathrm{P}_{0}$ on $S$, which corresponds to the value $w_{0}$ of the parmeter $w$ such that $n$ equations

$$
F_{\alpha}{ }^{\prime}\left(w_{0}\right)=0, \quad \alpha=1,2, \cdots, n,
$$

hold simultaneously, is a singular point of the surface, because all the coefficients of the first fundamental form of $S$ vanish at $w_{0}$.

Now suppose that $w_{0}$ corresponds to a singular point on $S$. We can assume without any loss of generality that $w_{0}=0$, because we can carry $w_{0}$ to 0 by a linear fractional transformation which carries the domain onto itself. We can assume without any loss of generality that $F_{\alpha}\left(w_{0}\right)=0$. So we have the following power series expansion of $F_{\alpha}(w)$ at the origin:

$$
F_{\alpha}(w)=c_{\alpha} w^{\lambda}+\cdots,
$$

where $\lambda(\geqq 2)$ is the least positive integer such that at least one of $c_{\alpha}$ 's does not vanish. By virtue of (1) we can see that $c_{\alpha}$ 's satisfy the relation

$$
\sum c_{\alpha}{ }^{2}=0 \text {. }
$$

On account of (5), we see that at least two of $c_{1}, c_{2}, \cdots, c_{n}$ do not vanish. So we assume that $c_{1} \neq 0$ and $c_{2} \neq 0$.

$1^{\circ}$. Suppose that

$$
c_{2}=k c_{1} \quad(k: \text { real })
$$

In this case, we can put

$$
c_{1}=r_{1} e^{i \theta}, \quad c_{2}=r_{2} e^{i \theta}
$$

where $r_{1}$ and $r_{2}$ and $\theta$ are real numbers. If we put

$$
\left\{\begin{array}{l}
F_{1} *=\frac{r_{1} F_{1}+r_{2} F_{2}}{\sqrt{r_{1}^{2}+r_{2}^{2}}}, \\
F_{2} *=\frac{-r_{2} F_{1}+r_{1} F_{2}}{\sqrt{r_{1}^{2}+r_{2}^{2}}}, \\
F_{\rho} *=F_{\rho} \quad(\rho=3,4, \cdots, n),
\end{array}\right.
$$

then $F_{\alpha} \rightarrow F_{\alpha}{ }^{*}$ is an orthogonal transformation. The power series expansion of $F_{\alpha}{ }^{*}$ has the same form as (4):

$$
F_{\alpha} *(w)=c_{\alpha} * w^{2}+\cdots
$$

However, we have

$$
c_{1} * \neq 0, \quad c_{2}^{*}=0 .
$$

As (8) is an orthogonal transformation

$$
\sum F_{\alpha}{ }^{* \prime 2}(w)=0
$$

holds good too. Substituting $F_{\alpha}$ and $F_{\alpha}{ }^{*}$ by $x_{\alpha}$ and $x_{\alpha}{ }^{*}$, we get an orthogonal transformation $x_{\alpha} \rightarrow x_{\alpha} *$. The equations

$$
x_{\alpha}{ }^{*}=\Re F_{\alpha}{ }^{*}
$$

are the equations of the minimal surface $S$ with respect to the new coordinate system $x_{\alpha}^{*}$.

$2^{\circ}$ Suppose that any relation of the type (6) does not hold. If $c_{3} \neq 0$, it is clear that there exist real constants $\lambda, \mu$ such that 
(12)

$$
c_{3}=\lambda c_{1}+\mu c_{2} .
$$

Now we consider an orthogonal transformation

$$
\left\{\begin{array}{l}
F_{1} *=\frac{-\mu F_{1}+\lambda F_{2}}{\sqrt{\bar{\lambda}^{2}+\mu^{2}}}, \\
F_{2} *=\frac{-\lambda F_{1}-\mu F_{2}-\left(\lambda^{2}+\mu^{2}\right) F_{3}}{\sqrt{\left(\lambda^{2}+\mu^{2}\right)\left(1+\lambda^{2}+\mu^{2}\right)}}, \\
F_{3} *=\frac{-\lambda F_{1}-\mu F_{2}+F_{3}}{\sqrt{1+\lambda^{2}+\mu^{2}}}, \\
F_{\rho} *=F_{\rho} \quad(\rho=4,5, \cdots, n),
\end{array}\right.
$$

then we can see that

$$
\begin{gathered}
F_{\alpha} *(w)=c_{\alpha} * w^{\lambda}+\cdots \\
c_{1} * 0, \quad c_{2} * 0, \quad c_{3} *=0 .
\end{gathered}
$$

Substituting $F_{\alpha}$ and $F_{\alpha}{ }^{*}$ by $x_{\alpha}$ and $x_{\alpha}{ }^{*}$, we get an orthogonal transformation $x_{\alpha} \rightarrow x_{\alpha}{ }^{*}$. The equations (11) where $F_{\alpha}{ }^{*}$ 's are given by (13) are the equation of $S$ with respect to the new coordinate system $x_{\alpha}$ *

$3^{\circ}$ Combining the processes explained in $1^{\circ}$ and $2^{\circ}$, we see that the equations of the minimal surface $S$ in a neighbourhood of its singular point can be brought to the form (2) having the condition (1), where $F_{\alpha}(w)$ 's are analytic functions on $W$ such that the power series expansions of $F_{\alpha}(w)$ in the neighbourhood of the origin have the form

$$
\left\{\begin{array}{l}
F_{1}(w)=c_{1} w^{2}+\cdots, \\
F_{2}(w)=c_{2} w^{\lambda}+\cdots, \\
F_{\rho}(w)=\quad c_{\rho} w^{\lambda+1}+\cdots \quad(\rho=3, \cdots, n)
\end{array}\right.
$$

where

$$
c_{1}^{2}+c_{2}^{2}=0 ; \quad c_{1}, c_{2} \neq 0
$$

If we put

$$
c_{1}=a_{1}+i b_{1}, \quad c_{2}=a_{2}+i b_{2},
$$

$a_{1}, b_{1}, a_{2}, b_{2}$ being real, then (17) can be written as

$$
a_{1}^{2}+a_{2}^{2}=b_{1}^{2}+b_{2}^{2}, \quad a_{1} b_{1}+a_{2} b_{2}=0 .
$$

Accordingly,

$$
\left\{\begin{array}{l}
F_{1}^{*}=\frac{b_{2} F_{1}-b_{1} F_{2}}{\sqrt{\bar{b}_{1}^{2}+b_{2}^{2}}}, \\
F_{2}^{*}=\frac{a_{2} F_{1}-a_{1} F_{2}}{\sqrt{\bar{a}_{1}^{2}+a_{2}^{2}}}, \\
F_{\rho} *=F_{\rho} \quad(\rho=3,4, \cdots, n)
\end{array}\right.
$$

is an orthogonal transformation.

We see that

$$
\left\{\begin{array}{l}
F_{1} *(w)=a w^{\lambda}+\cdots, \\
F_{2} *(w)=-i a w^{\lambda}+\cdots, \\
F_{\rho} *(w)=\quad c_{\rho} w^{\lambda+1}+\cdots \quad(\rho=3, \cdots, n)
\end{array}\right.
$$


where $a \neq 0$ is a real constant and $c$ 's are complex constants.

Substituting $F_{\alpha}$ and $F_{\alpha}{ }^{*}$ by $x_{\alpha}$ and $x_{\alpha}{ }^{*}$, we get an orthogonal transformation $x_{\alpha} \rightarrow x_{\alpha}{ }^{*}$. The equations of the from (11), where $F_{\alpha}{ }^{*}$ 's are given by (21), are the canonical form of the minimal surface $S$ in a neighbourhood of the singular point $\mathrm{P}_{0}$. We call a singular point which has the canonical from (21) a singular point of order $(\lambda-1)$.

\section{$\S 3$. The shape of a minimal surface in a neighbourhood of a singular point.}

Let us put $w=r e^{i \theta}$, then the equations (21) can be written as follows:

$$
\begin{array}{ll}
x_{1} & =a r^{\lambda} \cos \lambda \theta+\cdots, \\
x_{2} & =-a r^{\lambda} \sin \lambda \theta+\cdots, \\
x_{\rho} & =
\end{array}
$$

where [*] means a real constant whose value we do not need to know. The last equations give the minimal surface in consideration parametrically in terms of the parameters $(r, \theta)$.

If we differentiate the last equations by $r$, we have

$$
\begin{aligned}
& \frac{\partial x_{1}}{\partial r}=a \lambda r^{\lambda-1} \cos \lambda \theta+\cdots, \\
& \frac{\partial x_{2}}{\partial r}=-a \lambda r^{\lambda-1} \sin \lambda \theta+\cdots, \\
& \frac{\partial x_{\rho}}{\partial r} \quad[*] r^{\lambda}+\cdots .
\end{aligned}
$$

Suppose that $\theta=\theta_{0}, \theta_{0}$ fixed, and $r$ tends to zero, then the direction cosines of the tangent to the curve $\theta=\theta_{0}$ at $\mathrm{P}_{0}$ is given by

$$
\left(\operatorname{sgn} a \cdot \cos \lambda \theta_{0}, \operatorname{sgn}(-a) \cdot \sin \lambda \theta_{0}, 0, \cdots, 0\right) .
$$

Hence, the angle $\phi$ between two curves $\theta=\theta_{0}$ and $\theta=\theta_{1}$ at $\mathrm{P}_{0}$ is given by

$$
\cos \phi=\cos \lambda \theta_{0} \cos \lambda \theta_{1}+\sin \lambda \theta_{0} \sin \lambda \theta_{1}=\cos \lambda\left(\theta_{1}-\theta_{0}\right) .
$$

The last equation shows that at the origin of the $w$-plane and at its corresponding point $\mathrm{P}_{0}$ on the minimal surface, the mapping (21) is not conformal. The angle between two curves $\theta=\theta_{0}$ and $\theta=\theta_{1}$ on the minimal surface is $\lambda$ times of the angle between corresponding straight lines which issue from the origin on the $w$-plane. According to the terminology of the function theory our singular point of order $(\lambda-1)$ is a branch point of order $(\lambda-1)$.

\section{$\S 4$. Some differential geometric lemmas.}

Suppose that $x^{\alpha}=x^{\alpha}(u, v),(u, v) \in W$, is an arbitrary surface $S$ of class $C^{\prime \prime}$ in $E_{n}$. Then we have the Gauss' derived equation

$$
x_{, b c}^{\alpha}=\Omega_{b c}^{o} N_{\sigma}^{\alpha},
$$

where we have put $u=u^{1}, v=u^{2}$ and $x_{, b c}^{\alpha}$ 's are the covariant derivative of $x^{\alpha}{ }_{u b}$ 's with respect to the first fundamental tensor $g_{b c}, N_{\sigma}^{\alpha}$ 's are $(n-2)$ unit normal vectors of $S$ which are orthogonal to each other and $\Omega_{b c}^{a}$ is the second fundamental tensor of $S$ corresponding to $N_{\sigma}^{\alpha}$. 
In classical differential geometry we say that a surface which satisfies

$$
g^{b c} \Omega_{b c}^{a}=0 \quad(\sigma=1,2, \cdots, n-2)
$$

is a minimal surface.

LEMMA 1. A minimal surface in the sense of Weierstrass is a minimal surface in the sense of classical differential geometry, if the former has no branch points.

Proof. A minimal surface in the sense of Weierstrass is given by (2) with the condition (1). If we put $w=u^{1}+i u^{2}$ and

$$
F_{\alpha}=\phi_{\alpha}\left(u^{1}, u^{2}\right)+i \phi_{\alpha}\left(u^{1}, u^{2}\right),
$$

then we get

$$
x^{\alpha}=\phi_{\alpha}\left(u^{1}, u^{2}\right) .
$$

By virtue of the condition (1) we see that

$$
g_{11}=g_{22} \neq 0, \quad g_{12}=0 \text {. }
$$

Hence

$$
g^{11}=g^{22} \neq 0, \quad g^{12}=0 .
$$

Accordingly we see that the condition (24) reduces to

$$
\Omega_{11}^{\sigma}+\Omega_{22}^{\sigma}=0 .
$$

Therefore it is sufficient to prove

$$
x_{, 11}^{\alpha}+x_{, 22}^{\alpha}=0 .
$$

However, as we can easily verify them

$$
\begin{aligned}
& x_{{ }_{11}}^{\alpha}=x^{\alpha}{ }_{u^{1} u^{1}}-\frac{1}{2}\left(\log g_{11}\right)_{u^{1}} x^{\alpha} u^{1}+\frac{1}{2}\left(\log g_{11}\right)_{u^{2}} x^{\alpha} u^{2}, \\
& x_{{ }_{22}}^{\alpha}=x^{\alpha}{ }_{u^{2} u^{2}}+\frac{1}{2}\left(\log g_{11}\right)_{u^{1}} x^{\alpha} u^{1}-\frac{1}{2}\left(\log g_{11}\right)_{u^{2}} x^{\alpha}{ }_{u^{2}} .
\end{aligned}
$$

Hence, the condition (28) reduces to

$$
x^{\alpha} u^{1} u^{1}+x^{\alpha} u^{2} u^{2}=0 .
$$

This means that $x^{\alpha}$ 's are harmonic functions. However, $\phi_{\alpha}\left(u^{1}, u^{2}\right)$ 's are harmonic, our proof is complete.

Q.E. D.

Lemma 2. A minimal surface $S$ in the sense of classical differential geometry has non-positive Gaussian curvature.

Proof. The Gauss conditions of integrability of (23) are given by

$$
R_{a b c d}=\sum_{\sigma}\left(\Omega_{a d}^{\sigma} \Omega_{b c}^{\sigma}-\Omega_{a c}^{\sigma} \Omega_{b d}^{\sigma}\right)
$$

where $R_{a b c d}$ are Riemann-Christoffel's tensor of the first kind of $S$, i. e.

$$
R_{a b c d}=g_{a e} R_{b c d}^{e}
$$

where

$$
R_{b c d}^{e}=\frac{\partial\left\{\begin{array}{c}
e \\
b c
\end{array}\right\}}{\partial u^{d}}-\frac{\partial\left\{\begin{array}{c}
e \\
b d
\end{array}\right\}}{\partial u^{c}}+\left\{\begin{array}{c}
e \\
f d
\end{array}\right\}\left\{\begin{array}{c}
f \\
b c
\end{array}\right\}-\left\{\begin{array}{c}
e \\
f c
\end{array}\right\}\left\{\begin{array}{c}
f \\
b d
\end{array}\right\} .
$$

The Gaussian curvature of $S$ is given by every one of the following relations: 


$$
g_{11} K=R_{1_{1} \infty}^{\infty}, g_{22} K=R_{2_{21}}^{\sim}, g_{12} K=R_{21_{\infty}}^{\infty}=R_{121}^{1} .
$$

Now contracting $g^{a c} g^{b d}$ to (29), we get

$$
-g^{a c} R_{a c b}^{b}=g^{a c} g^{b d} \Omega_{a d}^{\sigma} \Omega_{b c}^{\sigma} \equiv \sum\left(\Omega^{\sigma}\right)^{2} .
$$

By virtue of (30), it is easily verified that the left hand side of the last equation is equal to $-2 K$. Hence we get

$$
K=-\frac{1}{2} \Sigma\left(\Omega^{\sigma}\right)^{2} \leqq 0
$$

Lemma 3. Let $x^{\alpha}(s)$ be a curve $C$ of class $C^{\prime \prime}$ on an arbitrary surface $S$ of class $C^{\prime \prime}$ in $E_{n}$. Then the curvature $k(s)$ of $C$ is not smaller than the geodesic curvature $k g$ of $C$ on $S$.

Proof. Let $u^{1}=u^{1}(s), u^{2}=u^{2}(s)$ be the equation of $C$ on the surface $S$ defined by $x^{\alpha}=x^{\alpha}\left(u^{1}, u^{2}\right)$. Then we get

$$
\begin{aligned}
\frac{d x^{\alpha}}{d s} & =\frac{\partial x^{\alpha}}{\partial u^{a}} \frac{d u^{a}}{d s}, \\
\frac{d^{2} x^{\alpha}}{d s^{2}} & =\frac{\partial^{2} x^{\alpha}}{\partial u^{a} \partial u^{b}} \cdot \frac{d u^{a}}{d s} \frac{d u^{b}}{d s}+\frac{\partial x^{\alpha}}{\partial u^{a}} \frac{d^{2} u^{a}}{d s^{2}} .
\end{aligned}
$$

By virtue of (23), the last equation can be written as

$$
\frac{d^{2} x^{\alpha}}{d s}=\left(\frac{d^{2} u^{a}}{d s^{2}}+\left\{\begin{array}{c}
a \\
b c
\end{array}\right\} \frac{d u^{b}}{d s} \frac{d u^{c}}{d s}\right) \frac{\partial x^{\alpha}}{\partial u^{a}}+\left(\Omega_{a b}^{\rho} \frac{d u^{a}}{d s} \frac{d u^{b}}{d s}\right) N_{\rho}^{a} .
$$

As the magnitude of

$$
\frac{d^{2} x^{\alpha}}{d s^{2}} \text { and } \frac{d^{2} u^{a}}{d s^{2}}+\left\{\begin{array}{l}
a \\
b c
\end{array}\right\} \frac{d u^{b}}{d s} \frac{d u^{c}}{d s}
$$

are $k(s)$ and the geodesic curvature $k_{g}(s)$ respectively, we get

$$
k^{2}(s)=k_{g}^{2}(s)+\sum_{\rho}\left(\Omega_{a b}^{\rho} \frac{d u^{a}}{d s} \frac{d u^{b}}{d s}\right)^{2} .
$$

The last equation shows that

$$
k(s) \geqq k_{g}(s),
$$

as $k(s)$ is non-negative always.

\section{$\S 5$. The main theorem.}

Let $x^{\alpha}(s)$ be the equation of a given simple closed curve $C$ of class $C^{\prime \prime}$ in $E_{n}$. According to the theory of J. Douglas on Plateau's problem, we can find analytic functions $F_{\alpha}(w)$ defined on the open unit circle $W$ so that they satisfy the relation (1) identically on $W$ and the mapping (2) of $W$ in $E_{n}$ has the following property: the boundary circle $\partial W$ is mapped homeomorphically upon the given closed curve $C$ by the mapping (2) in consideration.

The surface defined by (2) is a minimal surface of disk type bounded by the given closed curve $C$. It may have singular points in the sense of $\S 2$. However, singular points may exist only finite in number, because not all the functions are identically constant.

$1^{\circ}$ First we assume that there are no singular points on $S$. We subdivide the unit circle $W$ into a finite cell complex by smooth curves so that inner angles of cells 
are smaller than $\pi$. The mapping (2) is conformal as (25) shows it. The images of cells on $W$ by (2) constitute a subdivision of the minimal surface into a cell complex too. Applying Gauss-Bonnet's theorem to a cell $\sigma_{p}$ on the minimal surface we get

$$
\oint_{\partial \sigma_{p}} k_{g} d s+\iint_{\sigma_{p}} K d O+\Sigma(\pi-\phi)=2 \pi
$$

where $\phi$ 's are inner angles of $\sigma_{p}$. Summing up all these equations sides by sides we get

$$
\oint_{C} k_{g} d s+\iint_{S} K d O=2 \pi(e-k+f)
$$

where $e, k, f$ are numbers of vertices, sides and 2-cells of the subdivision of $W$ in consideration.

As $e-k+f=1$, we get

$$
\oint_{C} k_{g} d s+\ddot{\int} K d O=2 \pi
$$

However, as $S$ is a minimal surf́ace $K \leqq 0$, so we get, by virtue of (32)

$$
\oint_{S} k d s \geqq 2 \pi+\iint_{S}|K| d O .
$$

$2^{\circ}$ Next we assume that the minimal surface $S$ of disk type bounded by the given closed curve $C$ has just one singular point $\mathrm{P}_{0}$ of order $(\lambda-1)$. Denote the corresponding point on $W$ by $w_{0}$ and take a subdivision of $W$ into a finite cell complex such that it satisfies conditions described in $1^{\circ}$, and it has the point $w_{0}$ as a vertex and every cell which has a vertex at $w_{0}$ has an inner angle $<\pi / \lambda$ at $w_{0}$. Althought all coefficients of the first fundamental form of the minimal surface $S$ vanish at $w_{0}$, the point $\mathrm{P}_{0}$ on $S$ which corresponds to $w_{0}$ is not essentially a singularity for every cell on $S$ which corresponds to a cell on $W$ such that $w_{0}$ is a vertex. It is merely a singularity of the parametric representation. We can easily verify it by a parameter transformation

$$
\gamma^{2 \lambda}=\xi^{2}+\eta^{2}, \quad \tan \lambda \theta=\frac{\eta}{\xi} .
$$

Hence we may apply Gauss-Bonnet's theorem to every cell which corresponds to a cell on $W$ such that $w_{0}$ is a vertex.

If we sum up all equations of the type (33), then at $\mathrm{P}_{0}$ the term $\Sigma(-\phi)$ gives rise $-\lambda \cdot 2 \pi$ contrary to the value $-2 \pi$ of non-singular cases. Accordingly, we get

$$
\oint_{C} k d s \geqq 2 \pi+\iint_{S}|K| d O+2(\lambda-1) \pi .
$$

$3^{\circ}$ If the minimal surfaces $S$ has $m$ singular points of order $\left(\lambda_{1}-1\right), \cdots,\left(\lambda_{m}-1\right)$, then we can easily see that

$$
\oint_{C} k d s \geqq 2 \pi+\iint_{S}|K| d O+2 \sum_{i=1}^{m}\left(\lambda_{i}-1\right) \pi .
$$

Consequently, we get the following

Theorem. Let $C$ be a simple closed curve of class $C^{\prime \prime}$ in $E_{n}$. Then the total 
curvature of $C$ satisfies the inequality (34), where $S$ is the minimal surface of disk type bounded by $C$ and $\left(\lambda_{i}-1\right)$ 's are orders of branch points of the minimal surface.

If

$$
\oint k d s=2 \pi
$$

we can easily see that the minimal surface of disk type bounded by $C$ has no singular points and $K \equiv 0$. Hence, by virtue of (29) and (30) we get

$$
\sum_{\sigma}\left(\Omega_{a d}^{o} \Omega_{a c}^{\sigma}-\Omega_{a c}^{o} \Omega_{b d}^{a}\right)=0 .
$$

Putting $a=d=1, b=c=2$ and making use of (27), we see that

$$
-\sum_{\sigma}\left\{\left(\Omega_{11}^{\sigma}\right)^{2}+\left(\Omega_{12}^{\sigma}\right)^{2}\right\}=0
$$

from which we get

$$
\Omega_{b c}^{o}=0, \quad \sigma=1,2, \cdots, n-2 .
$$

The last relation shows that the minimal surface $S$ in consideration is a plane region. For a simple closed curve in a plane, it is evident that the equality (35) holds when and only when the curve is convex.

Q.E. D.

Fary and Milnor proved the conjectiure of Borsuk to the effect that "the total curvature of a knot in $E_{3}$ is grater than or equal to $4 \pi$ '”. I could not succeed to prove it exactly by my method, however it seems to be proved by showing that every knot can be bounded by a minimal surface of disk type which has at least a branch point. The thorem seems to be true for closed curves in spaces of constant negative curvature and in some other Riemanian spaces too.

\section{Mathematical Institute, Tôhoku University.}

\section{Literature}

[1] Fenchel W., Über Krümmung und Windung geschlossener Raumkurven. Math. Ann. 101 (1929), 238-252.

[2] Fenchel, W., On the differential geometry of closed space curves. Bull. Amer. Math. Soc. 57 (1951), 44-57.

[3] Liebmann, H., Elementarer Beweis des Fenchelschen. Satzes über die Krümmung geschlossener Raumkurven. Sitzber. Preuss Akad. Wiss. (1929), 392-393.

[4] Segre, B., Sui circoli geodetici di una superficie a curvatura totale costante, che contengono nell'interno una linea assegnata. Boll Unione Mat. Italiana 13 (1934) 279-283.

[5] Borsuk, K., Sur la courbure totale des courbes fermées. Ann. Soc. Polon. Math. 19 (1946), 251-265.

[6] Rutishauser, H., and H. Samelson, Sur la rayon d'une sphère dont la surface contient une courbe fermée. C. R. Paris 227 (1948), 755-757.

[7] Fary, M. I., Sur la courbure totale d'une courbe gauche faisant un noeud. Bull. Soc. Math. France 77 (1949), 128-138.

[8] Milnor, J. W., On the total curvature of knots. Ann. of Math. 52(1950), 248-257.

[9] Fox, R. H., On the total curvature of some tame knots. Ann. of Math. 52(1950), 258260.

[10] Douglas, J., Solution of the problem of Plateau. Trans. Amer. Math. Soc. 33(1931), 263-321. 